\title{
Dynamics of the solar active region finestructure
}

\begin{abstract}
B. Bovelet and E. Wiehr
Universitäts-Sternwarte, Geismarlandstraße 11, 37083 Göttingen, Germany

Received 22 May 2003 / Accepted 11 August 2003

Abstract. We investigate the dynamical behavior of the finestructure in a sunspot's surroundings and its penumbra from a speckle-reconstructed $60 \mathrm{~min}$ time series taken at the $45 \mathrm{~cm}$ Dutch Open Telescope (DOT) on La Palma. In the $1 \mathrm{~nm}$ spectral window containing the $G$-band, we determine the area of each feature and its time evolution by means of pattern recognition, particularly adapted to separate bright granular edges from inter-granular $G$-band bright points (BP). The evolution of each individual BP shows a stronger variation of the area than of the intensity. We analyze the horizontal motions of BP as a function of their distance from the sunspot center. Within a $6 \mathrm{Mm}$ ring around the outer sunspot border, most BP (4/5) move radially outwards; they are faster than the minority (1/5) of inward moving BP. The difference of both velocities indicates a radial outward drift which decreases from about $0.3 \mathrm{~km} \mathrm{~s}^{-1}$ at the outer penumbral border to zero at about $20 \mathrm{Mm}$ distance (28") from the sunspot center; a spatial range that we interpret as the extension of the sunpot "moat". This finding supports the idea of giant rolls in deep layers measured by helio-seismic tomography and predicted by theory. Inside the penumbra, we find a $4 / 5$ majority of penumbral bright structures (PBS) to move inwards with a mean velocity of $0.8 \mathrm{~km} \mathrm{~s}^{-1}$. The $1 / 5$ minority of outward moving PBS is almost entirely located in the outer penumbra; their mean velocity of $0.8 \mathrm{~km} \mathrm{~s}^{-1}$ is equally found for penumbral dark structures (PDS) in the outer penumbra, in agreement with penumbral MHD models.
\end{abstract}

Key words. Sun: sunspots - Sun: magnetic fields - Sun: helioseismology

\section{Introduction}

Sunspots are known to be surrounded by a "moat" region (Sheeley 1969) with a preferable outward drift of solar structures such as "moving magnetic features" (Vrabec 1971) or EUV $1070 \AA$ A continuum brightenings (Georgakilas et al. 2002). These manifest in the "solar filigree" (Dunn \& Zirker 1973), being in continuum images only visible at very high spatial resolution. In $G$-band filtergrams, however, they are already visible at slightly lower resolution (see Muller \& Roudier 1984) as " $G$-band bright points" (BP). According to Langhans et al. (2002), these BP cover two families: (i) enhanced granular edges with upward velocities, and (ii) magnetic features with downward velocities. The latter are foot-points of the kiloGauss magnetic fields, detected with line-ratio measurements (Stenflo 1973) and complemented with a "slit-ratio" method at high spatial resolution (Wiehr 1978).

The radial outward drift in the sunspot moat may be caused by giant rolls, recently detected by helio-seismic tomography deep beneath the solar surface (Gizon et al. 2000; Zhao et al. 2001), and predicted by MHD models (Hurlburt \& Rucklidge 2000). The signature of such deeply-rooted motions at the solar surface will be masked by the faster stochastic granular motions. A separation of both requires sufficiently large statistics and thus an automatic pattern recognition particularly able to

Send offprint requests to: E. Wiehr, e-mail: ewiehr@uni-sw.gwdg.de separate the two families (i) and (ii) mentioned above. Such a distinction may also be useful to investigate the striking discrepancy between calculated and observed BP intensity contrasts reported by Sánchez Almeida et al. (2001). They argue that a possible relation between area and intrinsic brightness of BP may be masked by insufficient spatial resolution. It is thus worthwhile to investigate a possible "intensity-area relation" from high resolution images.

\section{Observations}

We use a one hour time series of active region AR 9407 at $11^{\circ} \mathrm{N} / 21^{\circ} \mathrm{E}$ obtained with the Dutch Open Telescope (DOT) on La Palma on April 1, 2001. The time lapse between successive images is $0.5 \mathrm{~min}$; one pixel corresponds to 0.071 (i.e., $51.5 \mathrm{~km}$ on the sun), the images cover about $83^{\prime \prime} \times 61^{\prime \prime}$. The speckle masking technique (Weigelt 1977; von der Lühe 1984) was applied to remove influences from seeing, using the computer code from de Boer (1996) after improvements by P. Sütterlin (cf. Sütterlin \& Wiehr 1998): In particular, the artificial distortions in the reconstructed images, arising from segmentation into small iso-planar patches, are avoided by a correlation procedure referring to the temporal mean image (Sütterlin, private communication). Besides, such effects will hardly interfere since we assume a vector of constant, linear velocity fitted to the feature's center motions. An example is shown in Fig. 1; the spatial resolution of reconstructed DOT images may reach 0.'2 (cf. Sütterlin 2001). 


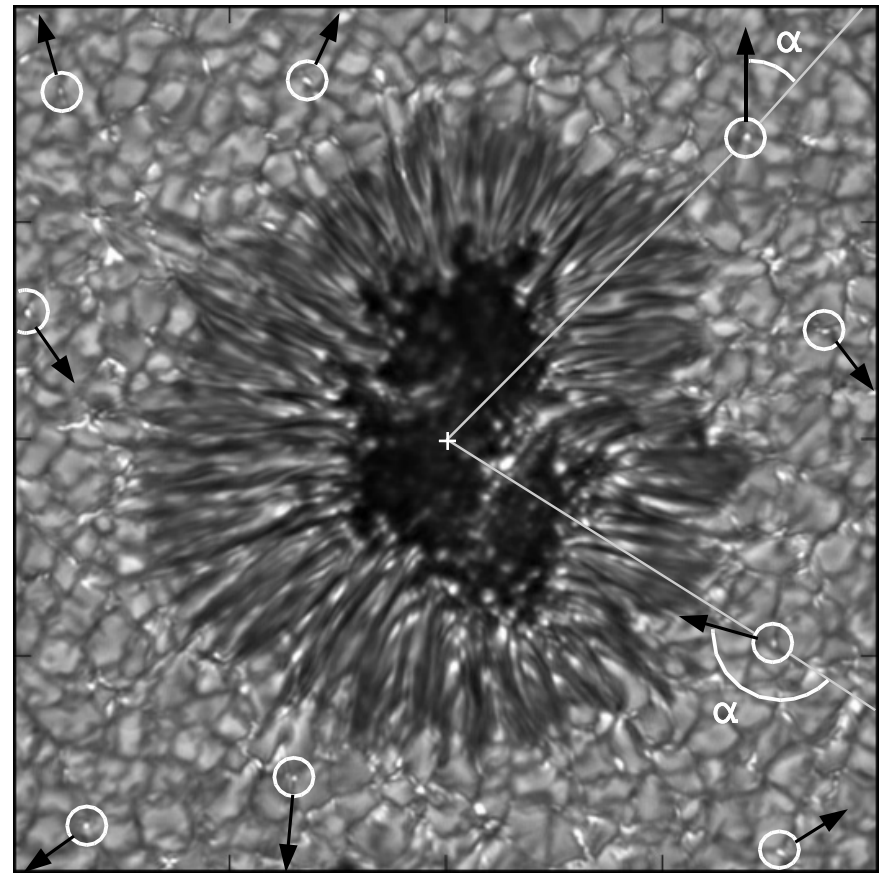

Fig. 1. Central sunspot area, $45^{\prime \prime} \times 45^{\prime \prime}$, of the speckle reconstructed $G$ band image in the mid of the $60 \mathrm{~min}$ time series for AR-9407 at $11^{\circ} \mathrm{N} /$ $21^{\circ} \mathrm{E}$; some isolated BP are encircled with their respective velocity vectors; for two of them the vectors' radial position angle with the spot radius vector is indicated, $0^{\circ} \leq \alpha \leq 180^{\circ}$ either clockwise or counter-clockwise yielding $\alpha=0^{\circ}$ for radial outward and $\alpha=180^{\circ}$ for radial inward velocities.

\section{Pattern recognition procedure}

In a previous paper (Bovelet \& Wiehr 2001; hereafter referred to as Paper I), we described a powerful method of pattern recognition using a "multiple level tracking" algorithm (MLT), and applied it to solar granulation and limb faculae. This algorithm starts a detection of structures at high intensity levels and repeatedly extends them to lower levels, thus filling more and more of the observed intensity contours. At each brightness threshold, the algorithm additionally includes new shapes appearing for the first time, but does not allow at low levels contact of neighboring shapes which are distinct at higher levels. Thus, chains of closely packed BP (forming the filigree "crinkles"; Dunn \& Zirker 1973) are separated into single members, assuming that the spaces between them appear less dark due to the limited spatial resolution. A similar approach was used in Paper I for closely neighboring granules and resulted in the finding of a self-similarity of granules with a constant fractal dimension of 1.15 independent of their area sizes.

If we apply our algorithm to the morphology of $G$-band bright points (BP), we have to add an additional criterion for a separation of the two BP families detected by Langhans et al. (2002). Since we are only interested in BP which are manifestations of inter-granular flux-tubes, we have to ignore those $\mathrm{BP}$ which represent bright granular edges. This is done by a particular contrast criterion (applied additionally to MLT) for which we use the higher contrast of the (magnetic) BP family (ii) to their inter-granular surroundings: we select the darkest $50 \%$ of pixels closely adjacent to a feature selected by MLT,

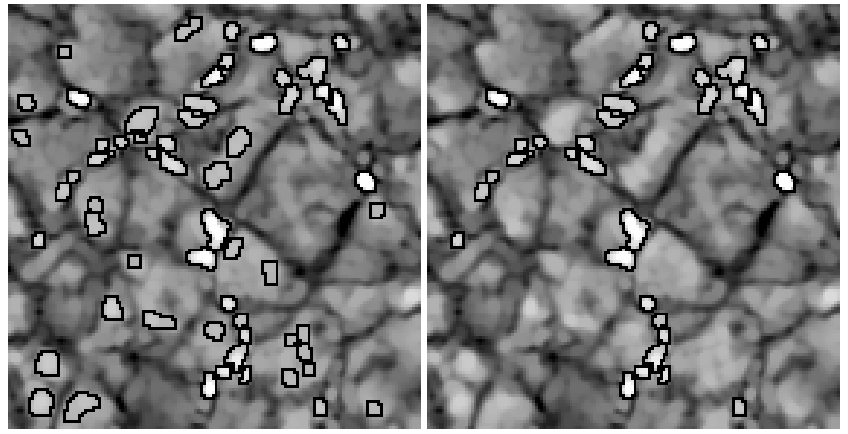

Fig. 2. Pattern recognition at different contrast thresholds; left panel: pure "multi-level-tracking" MLT; right panel: MLT plus additional "local contrast selection" eliminating bright granular edges.

and determine their ratio with the brightest $50 \%$ of pixels within the feature's area. With this additional criterion, our algorithm succeeds in separating the desired inter-granular features from intrinsic granular brightenings (Fig. 2).

Our pattern recognition method differs from other methods since it returns a realistic approximation of the observed photometric morphology even for elongated shapes with complex contours. A pure brightness criterion would depend to a considerable extent on the intensity distribution within a structure which, in turn, depends on the image quality varying with time even in series of reconstructed images. Since the algorithm iteratively extends each recognized feature to a realistic shape at the basic MLT level, the areas obtained are highly suitable to determine the $f l u x$ - given by the sum of the intensities of all the pixels within the outermost contour - which is largely independent of remaining variations of the image quality.

For the exact position of each BP, we take the "center-ofgravity" of each individual shape, and use it as a tracer for the determination of dynamical quantities as, e.g., horizontal motion. We identified and tracked a total of 40246 features in the 120 images of the $60 \mathrm{~min}$ time sequence of active region AR-9407. Among these, we finally select 11384 features representing sequential appearances of 1426 individual BP which are isolated, i.e. neither split nor merge during their lifetime periods.

\section{Results for G-band bright points}

\subsection{Temporal evolution of $B P$}

For a realistic determination of the evolution of a "typical" BP we trace it forward and backward in time until its brightness falls below the lowest intensity threshold ("basic MLT level"; cf. Paper I) used for shape detection by our algorithm. The choice of that level does not essentially affect our results since the structures under study show rather steep intensity gradients at their edges. We thus follow the evolution of a total of 1426 single BP. Each of them is numerically tagged and sequentially tracked through a part of the total field of view centered on the particular BP. An example for the evolution of a BP is given in Fig. 3 where the selected feature is whitened (and neighbored ones set to gray). 


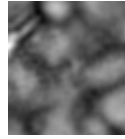

$24^{\prime \prime} 0^{\prime \prime}$

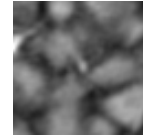

$24^{\prime} 30^{\prime \prime}$
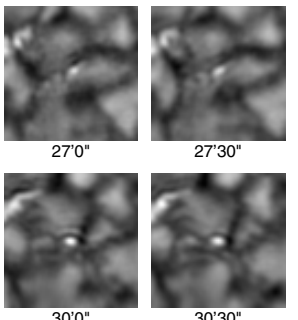
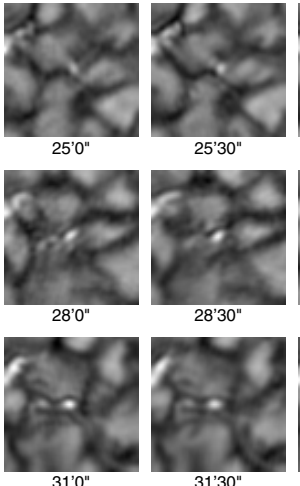

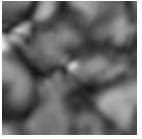

$260^{\prime \prime}$
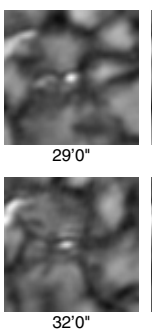
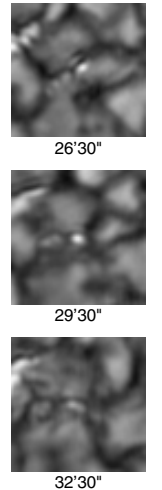
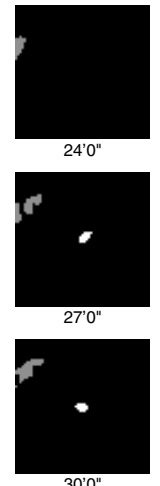

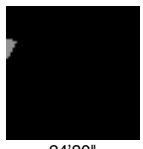

$24^{\prime} 30^{\prime \prime}$
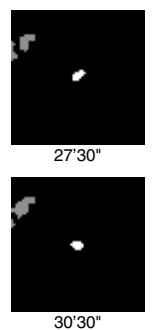

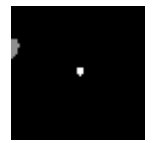

$25^{\prime} 0^{\prime \prime}$
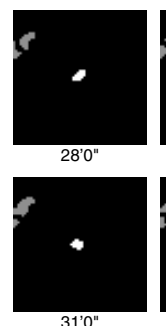
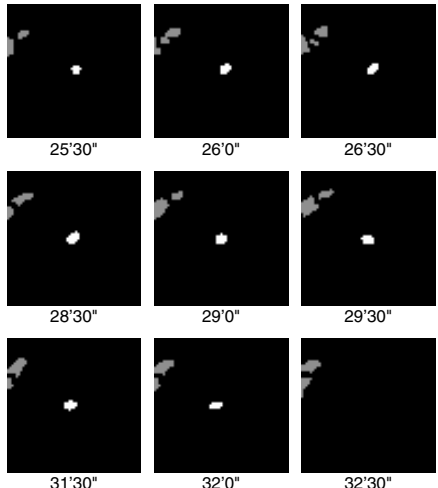

Fig. 3. Evolution of a $G$-band bright point in sequential frames (left panel) together with the binary maps of the MLT pattern recognition algorithm (right panel); the selected shape is whitened, neighboring features set to gray; boxes show 4 ". $25 \times 4$.'. 25 sub-fields.

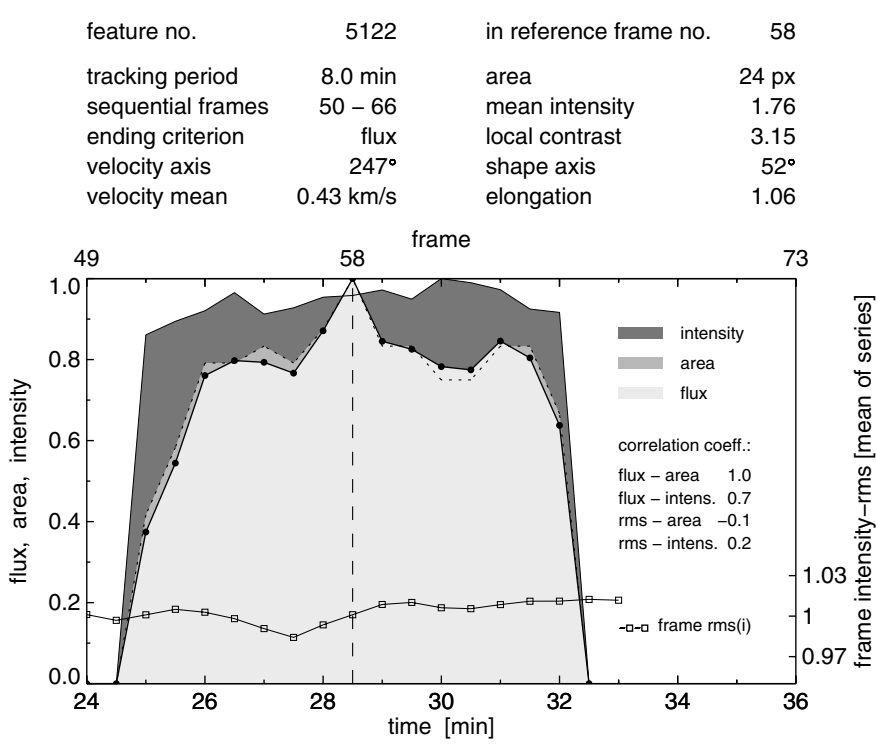

Fig. 4. Evolution of flux, area, and mean intensity of the selected $G$ band feature from Fig. 3 scaled on its maximum values; for comparison the time variation of the intensity fluctuation (rms) in each frame. The table at the top gives parameters automatically determined by the algorithm; in the left column for the evolution, in the right column for the morphology at maximum flux; "mean intensity" in units of the photospheric level around the spot.

The temporal evolution of the selected BP (whitened in Fig. 3) is shown in Fig. 4 by its characteristic parameters area (pixel), flux, and mean intensity normalized to their maximum values. The header of Fig. 4 gives characteristic properties automatically returned by the algorithm for each BP as selected by MLT and tracked through its visibility period: dynamical parameters (left part of the header) are derived from feature tracking; morphological characteristics (right part) are found at the moment of maximum flux ("reference frame").

Figure 4 shows that the time evolution of a BP is dominated by the area which varies up to a factor of 5 , whereas the intensity varies only between 0.8 and 1.0. This behavior does not arise from temporally varying image quality, as is seen from the intensity rms. This is also confirmed in Fig. 5, which gives the mean values of intensity and area for all recognized features in each frame through the entire time series together with the intensity-rms of each frame (given by the average intensity fluctuation in units of the frame's mean intensity). It can be seen that brightness and area are highly correlated (0.9), but no correlation is found with the intensity-rms. The lower curve of Fig. 4 reflects the same finding. It demonstrates that the "intensity-area relation" of BP is not caused by the image quality.

\subsection{Intensity - area relation of single $B P$}

We investigate the "intensity-area relation" for our 1426 isolated BP. Figure 6 shows a two-dimensional histogram of their mean intensity (in units of the mean intensity outside the spot, $I_{\text {phot }}$ ) and of their area at the moment of their maximum flux, i.e. independent from each BP's temporal evolution. The number density in this histogram is visualized by iso-contour lines (at the top plane in Fig. 6). We determine the intensity-area relation by fitting a polynomial of degree 3 to the mean intensities per area interval of 4 pixel bin-area and find an increase of intensity with area (i.e. the "ridge" of the number density "mountain" in Fig. 6). Near the most frequent intensity $I=1.5 \times I_{\text {phot }}$, this relation is characterized by a linear section with a gradient of $\Delta I=0.1 \times I_{\text {phot }}$ per $40000 \mathrm{~km}^{2}(15 \mathrm{px})$; for larger areas the features reach a limit of $I \approx 1.7 \times I_{\text {phot }}$.

Interestingly, the same slope is found for the 11384 appearances (features) of the $1426 \mathrm{BP}$ through their life periods in sequential frames (Fig. 7). Hence, the temporal variation during the lifetime of each BP reflects the same intensity-area relation as the entity of BP at their maximum evolution. The relation is similar to the one found by Berger et al. (1995) but at a somewhat smaller scatter. Our mean BP intensities are higher than (even) the peak intensities given by Berger et al. (1995), since the speckle reconstruction strongly enhances small spatial scales.

\subsection{Dominant area of $B P$}

In order to get an idea about the "typical diameter" of BP, we refer to the 1426 isolated BP (i.e., unaffected by merging or splitting) and consider that BP are not a priori of circular shape. Our recognition of areas in units of pixels allows us to 


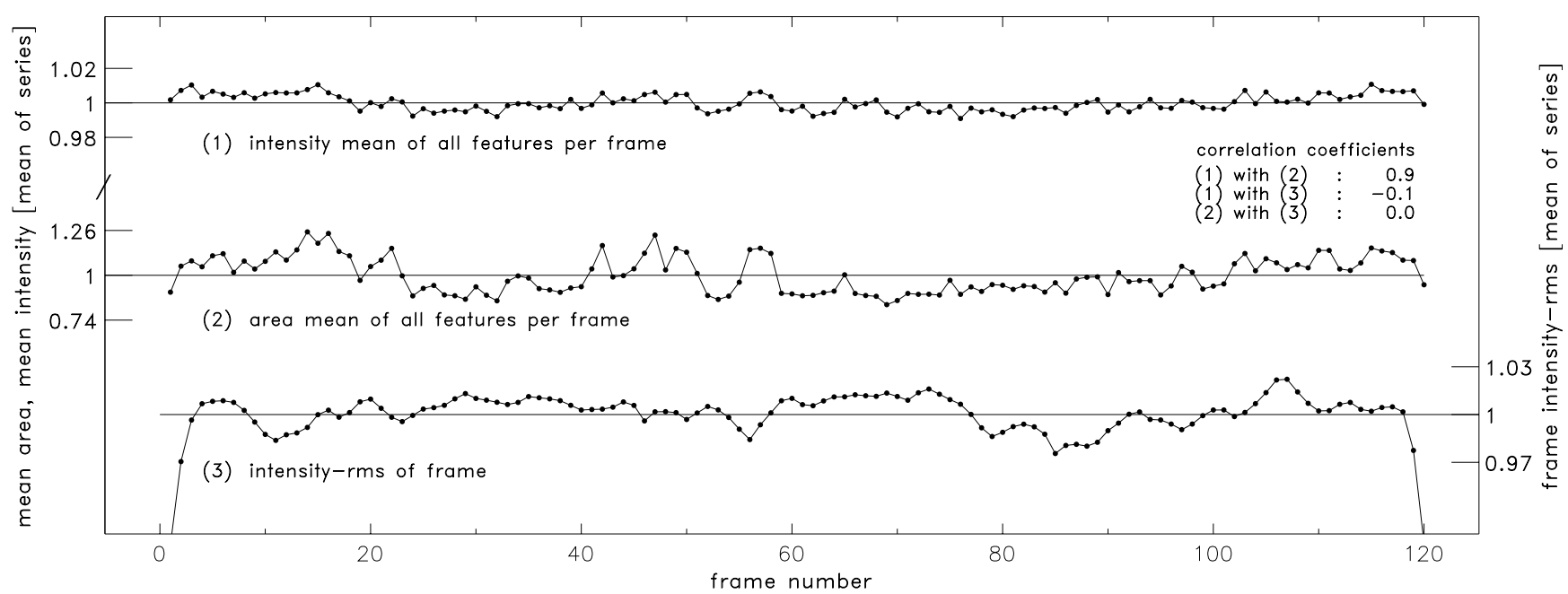

Fig. 5. Temporal variation of mean intensity and mean area of all isolated BP in each frame, correlated with the average deviation (rms) of all pixel intensities from the mean value of the entire sequence, indicating the image quality through the 120 frames.

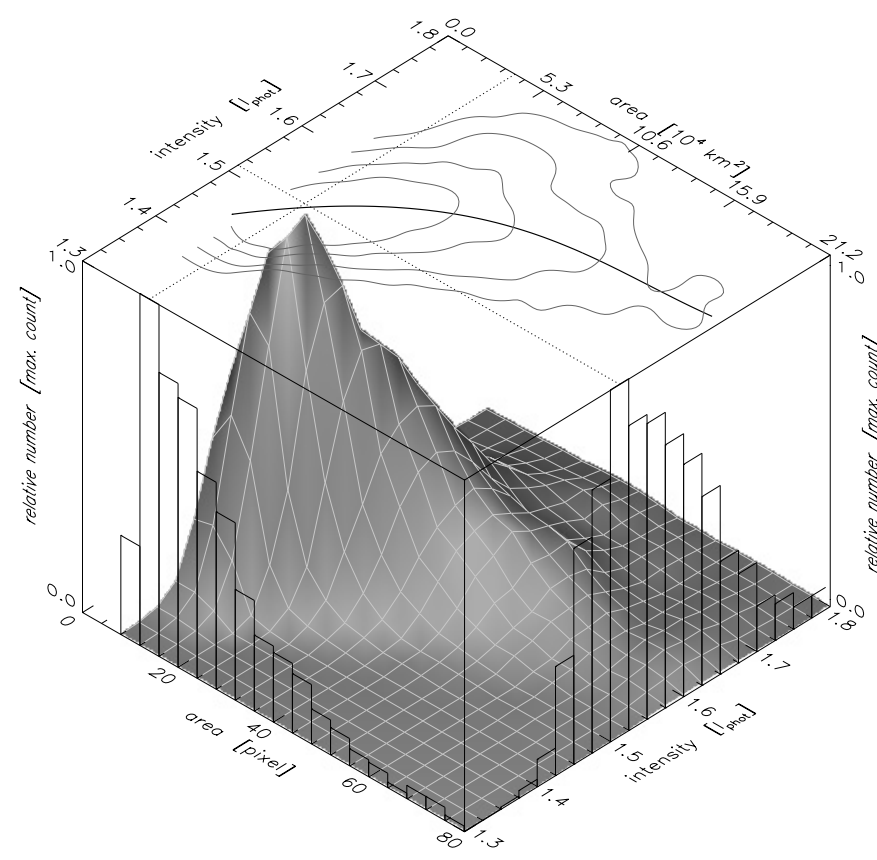

Fig. 6. Two-dimensional histogram of area and intensity of $1426 \mathrm{BP}$ at maximum flux, i.e. independent of each BP's temporal evolution. Intensity in units of $I_{\text {phot }}$; area in pixels; the upper plane shows contours of the 2D-histogram and a polynomial fit (degree 3 ) of the mean intensity per area-bin (4 px), giving the "intensity-area relation" for BP.

determine the real shape of a BP. We fit an ellipse to the assembly of pixels covering a BP such that the areas are equal. The ratio of both ellipse axes shows that about $2 / 3$ of the isolated BP are of circular shape with a dominant diameter of $4.2 \pm 0.5$ pixel-widths, i.e., $220 \pm 25 \mathrm{~km}$ on the sun; both in agreement with Berger et al. (1995). Since this extension (of 0. 3) is close to DOT's resolution (cf. Sütterlin 2001), the actual size of the BP may be smaller. The 4.2 pixel value corresponds to a circular shape covering a 14 pixel area which, indeed, dominates the area histogram Fig. 6 and thus confirms that BP are preferably circular.

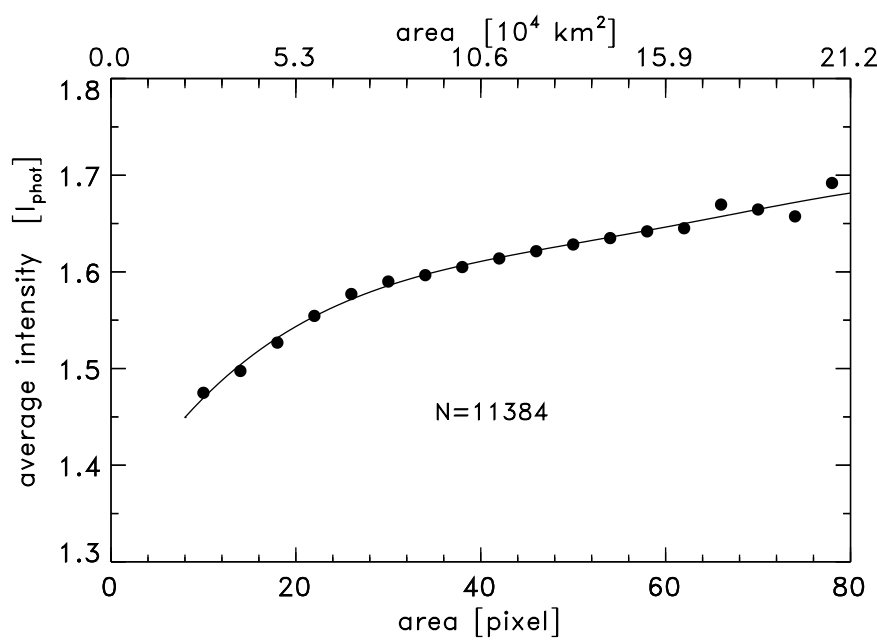

Fig. 7. "Intensity-area relation" for 11384 features representing the temporal evolution of the 1426 isolated BP tracked over their lifetimes in sequential frames of AR-9407.

\subsection{Horizontal motion of $B P$}

Our time series of speckle-reconstructed images allow us to describe the motion of BP on the solar surface, using for each feature the sequential displacement of its geometrical center. A linear fit over its detection period yields the vector of the mean velocity for each BP. As a side-product, we find that isolated $B P$ are slightly faster than non-isolated $B P$, possibly due to the processes of merging and/or splitting.

If we consider only the 1426 isolated BP, we find a total of 806 BP located farther away from the spot which show stochastic motions (Fig. 8a). Here, we obtain a mean velocity of $0.85 \mathrm{~km} \mathrm{~s}^{-1}$ which close to the value by Nisenson et al. (2003) and slightly slower than the $\approx 1.0 \mathrm{~km} \mathrm{~s}^{-1}$ found by various authors farther away from a spot (cf. Georgakilas et al. 2002 and references therein); it is slightly higher than the $0.65 \mathrm{~km} \mathrm{~s}^{-1}$ found by Muller \& Mena (1987) for facular points.

However, in a $6 \mathrm{Mm}\left(8^{\prime \prime} .3\right)$ wide ring around the sunspot, we find 284 BP of which $4 / 5$ move preferentially away (Fig. 8b) whereas $1 / 5$ move toward the sunspot, the mean of both values 

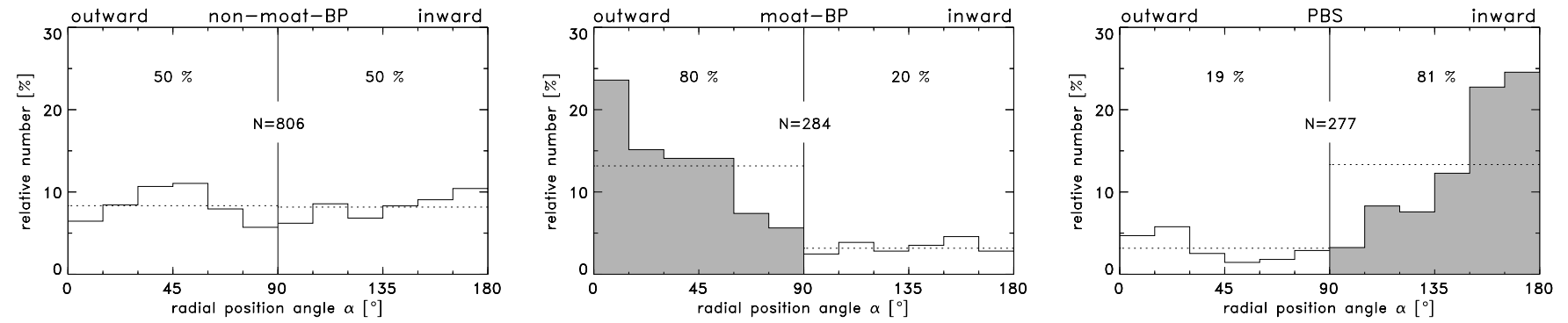

Fig. 8. Motion of 806 isolated BP outside $20 \mathrm{Mm}$ from the spot center (left), 284 isolated BP within a $6 \mathrm{Mm}$ wide ring around the spot (middle), and 277 penumbral bright structures (right panel); $\alpha$ is the angle between the velocity vector (cf. Fig. 1) and the radial direction to the spot center; $\alpha=0^{\circ}$ for radial outward, $\alpha=180^{\circ}$ for radial inward velocities; dotted lines give assumed equi-distributions separately for inward and outward motions and show that radial directions dominate.
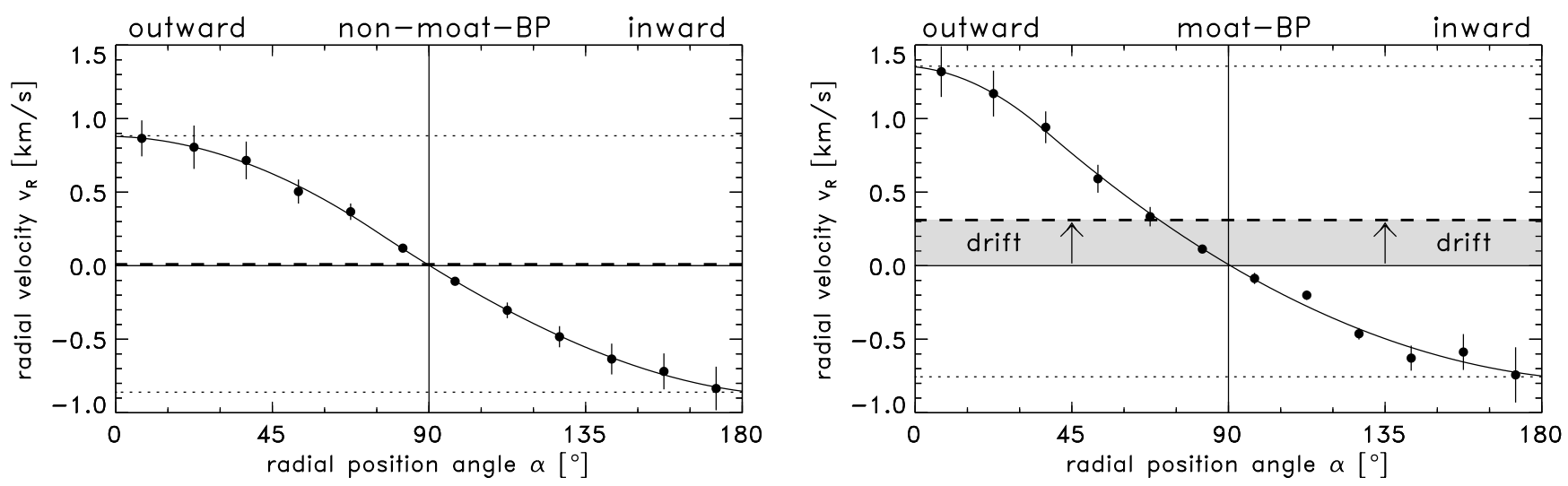

Fig. 9. Radial component of the mean velocity vector for the BP outside the moat ("non-moat BP"; left) and the BP within a $6 \mathrm{Mm}$ wide ring around the spot edge ("moat BP"; right panel); $\alpha$ as in Fig. 8; the underlying drift is indicated by the mean value of the anti-parallel velocities at $\alpha=0^{\circ}$ and at $\alpha=180^{\circ}$.

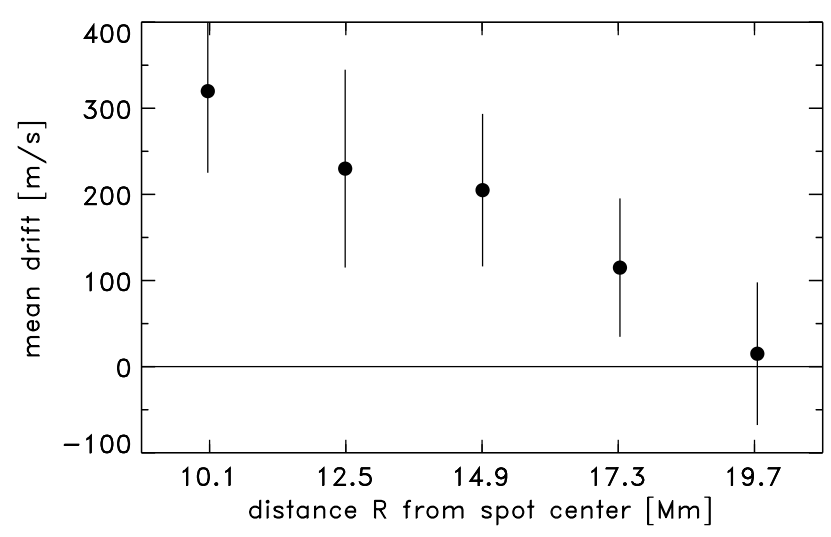

Fig. 10. Radial variation of the drift in the (circularly averaged) moat with distance $R$ from the spot center (upper panel); zero drift at $R \approx 20 \mathrm{Mm}\left(28^{\prime \prime}\right)$ defines the outer moat boundary. The error bars are deduced from the standard deviations of the mean velocities in Fig. 9 for each concentric ring (width $2.4 \mathrm{Mm}$ ) around the spot.

being close to the mean velocity found outside the moat. The accumulation of velocity vectors towards the radial outward direction ( $\alpha=0^{\circ}$; cf. left side of Fig. 8b) suggests an aligning force, resembling the known radial drift in the sunspot moat (see introduction), which does not occur in the stochastic motions farther away (Fig. 8a).

Since that drift is included in the above total velocity means (averaging over all directions for both, inward and outward motions), we may separate it from superposed stochastic motions by measuring the velocity difference between the outward and the inward moving features of isolated BP. For this purpose, we plot in Fig. 9 the amount of each velocity vector's radial component $V_{R}$ versus the radial position angle $\alpha$ of the velocity vector's direction; for inward motions $\left(90^{\circ}<\alpha \leq 180^{\circ}\right)$ we use negative values $V_{R}$. Any underlying radial drift should now appear as an asymmetry between the amounts of the (maximum) radial components for outward $\left(\alpha=0^{\circ}\right)$ and inward $\left(\alpha=180^{\circ}\right)$ velocities.

Far away from the sunspot, the amounts of outward and inward velocities are, as expected, quite similar (Fig. 9a), thus indicating no systematic drift. In a $6 \mathrm{Mm}\left(8^{\prime \prime} .3\right)$ vicinity to the penumbral edge, however, such an asymmetry with respect to the $V_{R}=0$ line is evident (Fig. 9b). Extrapolating to the anti-parallel directions of both, radially outward $\left(\alpha=0^{\circ}\right)$ and inward $\left(\alpha=180^{\circ}\right)$ motions, we find absolute values of $1.35 \mathrm{~km} \mathrm{~s}^{-1}$ for the outward and $0.75 \mathrm{~km} \mathrm{~s}^{-1}$ for the inward velocities. Half of the difference reveals the contribution by a mean radial drift of about $0.3 \mathrm{~km} \mathrm{~s}^{-1}$.

In order to determine the variation of that drift with radial distance $R$, we apply the above procedure in eight concentric rings of $\Delta R=1.8 \mathrm{Mm}$ width $\left(2.5^{\prime \prime}\right)$, and find a decrease to zero at $R \approx 20 \mathrm{Mm}$ from the sunspot center (28" ; Fig. 10). We verified that this radial decrease does not depend on the spatial binning, i.e. neither on the widths nor on the number of the concentric rings. The $20 \mathrm{Mm}$ distance represents a mean outer 


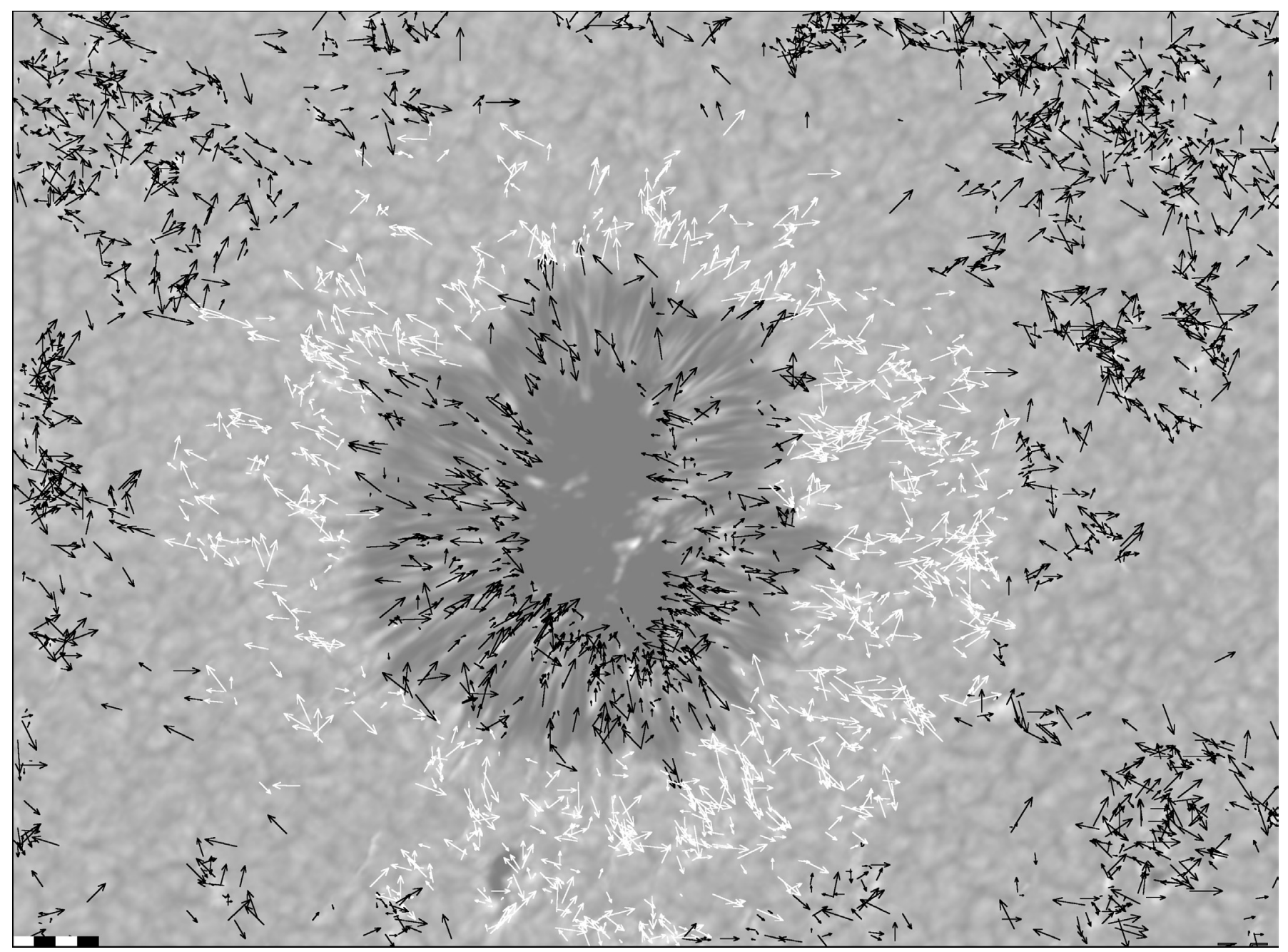

Fig. 11. Horizontal velocity vectors of sub-arcsec solar structures in penumbra and "moat" of a sunspot: 929 penumbral bright structures (inner black arrows) and $3682 \mathrm{G}$-band bright points in the spot "moat" (white arrows) and farther away (outer black arrows) plotted with an intensity average through the whole time series; scale in the lower left: $1 \mathrm{~km} \mathrm{~s}^{-1}$ per unit.

boundary, which we may readily use to define the outer border of the sunspot "moat", as visualized in Fig. 11.

\section{Penumbral structures}

We also apply our MLT algorithm to Penumbral Bright Structures (PBS), which may be conglomerates of smaller penumbral grains (cf. Berger et al. 2003). Optimizing the threshold levels for the penumba, MLT finds a total of 929 PBS and selects 277 isolated ones that neither merge nor split through the time sequence. We find that $4 / 5$ of the PBS move inwards toward the umbra (Fig. 8b) and are mostly located in the inner penumbra (see Fig. 11). This behavior agrees with the known one of bright penumbral grains. Our 4:1 ratio between the number of inwards and outwards moving PBS is slightly larger than that of Sobotka et al. (1999), and is much larger the $1: 1$ relation found by Sobotka \& Sütterlin (2001). It is in accordance with Schlichenmaier's (1998) penumbral model of rising flux-tubes which predicts a predominant inward motion at the inner penumbral boundary.
The velocity distribution in the penumbra is rather asymmetric with a "tail" to larger velocities: we find a mean of 0.8 , a median of $0.6 \mathrm{~km} \mathrm{~s}^{-1}$. The median of $0.6 \mathrm{~km} \mathrm{~s}^{-1}$ is close to that of $0.5 \mathrm{~km} \mathrm{~s}^{-1}$ found by various authors (cf. Sobotka \& Sütterlin 2001 , and references therein). We do not find systematic velocity variations along the radial spot direction, in contrast to Muller (1976) and to Denker (1998), but in agreement with Toenjes \& Wöhl (1982).

Whereas the inner penumbra shows well organized radial inward motions, PBS near the outer penumbral boudary are less organized and show both outward and inward motions (see Fig. 11) in agreement with Toenjes \& Wöhl (1982) and with Denker (1998). Due to temporal variations of the (irregular) outer spot boundary, it is difficult to decide whether a structure represents a BP (outside the spot) or a PBS or a transient feature; all of them showing similar velocities.

We tried to verify this behaviour also for Penumbral Dark Structures (PDS). For this purpose, we inverted the images: the then bright PDS are easily recognized by our MLT algorithm. We find that the number of outward moving PDS exceeds that of the inward moving PDS; this is in contrast to the PBS which 
preferably move inwards. The outward motion of both, PDS and outer PBS, might be the signature of the "wave-like" outwards drifting flux-tubes modelled by Schlichenmaier (2002). Each of these 'sea serpents' intersects the $\tau=1$ surface twice in the outer penumbra: their inner footpoint is bright and might affect the outwards moving PBS; their dark outer footpoint is dark and might affect the outwards moving PDS.

Structures which pass the outer spot border must be of completely different origin than the known Evershed effect, the spectral signature of which being a strong line asymmetry which ceases abruptly at the outer penumbral edge (see, e.g., review by Wiehr 1999). The additional small Doppler shifts of the line-cores of $1-2 \mathrm{~km} \mathrm{~s}^{-1}$, occasionally measured outside the spot border (e.g., Börner \& Kneer 1992) might be connected with the granular motions superposing our $0.3 \mathrm{~km} \mathrm{~s}^{-1}$ moat drift. The velocity packets travelling from the penumbra into its surroundings with velocities up to $5 \mathrm{~km} \mathrm{~s}^{-1}$ (Rimmele 1994) largely exceed our drift of $0.3 \mathrm{~km} \mathrm{~s}^{-1}$.

\section{Conclusions}

Among the two BP classes found by Langhans et al. (2002), we investigate in this paper exclusively those located in intergranular lanes, as our algorithm skips bright granular edges. According to Langhans et al. (2002), the thus selected BP are the signatures of magnetic concentrations. They may trace numerous disorganized magnetic concentrations (e.g., the MISMAs proposed by Sánchez Almeida \& Lites 2000, or the structures in the numerical simulations by Cattaneo 1999). Such magnetic concentrations may also produce $G$-band BP (see Steiner et al. 2001).

Interestingly, the time evolution of a BP's intensity flux is mostly affected by the area; we verified that this is not caused by varying image quality. It differs from the measured decrease of intensity with increasing area in the continuum (Keller 1992). This might reflect a basic difference between the origin of the $G$-band and the continuum intensity. The amount of the drift decreases from $0.3 \mathrm{~km} \mathrm{~s}^{-1}$ at the penumbral edge to zero at a $20 \mathrm{Mm}$ radial distance from the sunspot center $\left(28^{\prime \prime}\right)$, which we consequently define as the boundary of the sunspot moat.

The drift might also continue slightly inside the penumbra where several structures move over the outer sunspot border. Our finding favors the idea of deeply rooted giant flow systems located beneath the moat. Since we obtain a drift maximum near the spot border, we cannot exclude that the giant cell also affects motions of features in the outer penumbra (Fig. 12). This would agree with helio-seismic results by Gizon et al. (2000) and Zhao et al. (2001). We suggest that the magnetic concentrations forming the BP might be rooted deeply enough to be advectively driven by large-scale motions, and are additionally affected by random small-scale granular motions higher up in the atmosphere.

Acknowledgements. We are indebted to Dr. P. Sütterlin who put his observations from the Dutch Open Telescope, DOT, at our disposal and applied to them his improved speckle masking procedure. Drs. J. Sanchez-Almeida and R. Schlichenmaier contributed helpful suggestions; Dr. F. V. Hessman critically reviewed the manuscript.

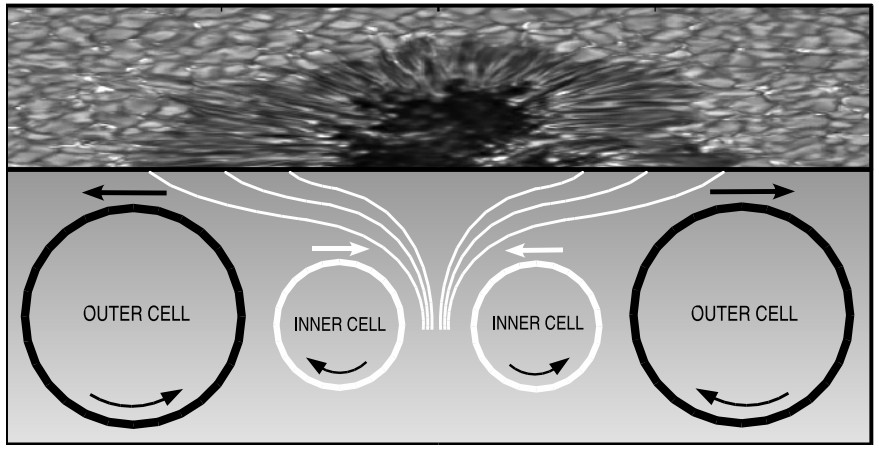

Fig. 12. Sketch of the large convective cell motions beneath a sunspot, predicted by theory to bundle the magnetic field-lines, and recently detected by helio-seismology.

\section{References}

Berger, T. E., Shrijver, C. J., Shine, R. S., et al. 1995, ApJ, 454, 531 Berger, T. E., Löfdahl, M. G., Scharmer, G., \& Title, A. M. 2003, AAS, SPD 34, 1108

Börner, P., \& Kneer, F. 1992, A\&A, 255, 307

Bovelet, B., \& Wiehr, E. 2001, Sol. Phys., 201, 13

Cattaneo, F. 1999, ApJ, 515, L39

de Boer, C. R. 1996, A\&AS, 120, 195

Denker, C. 1998, Sol. Phys., 180, 81

Dunn, R. B., \& Zirker, J. B. 1973, Sol. Phys., 33, 281

Georgakilas, A. A., Muglach, K., \& Christopoulou, E. B. 2002, ApJ, 576,561

Gizon, L., Duvall, T. L. Jr., \& Larsen, R. M. 2000, J. Astrophys. Astr., 21,339

Hurlburt, N. E., \& Rucklidge, A. M. 2000, MNRAS, 314, 793

Keller, C. U. 1992, Nature, 359, 307

Koutchmy, S. 1977, A\&A, 61, 397

Langhans, K., Schmidt, W., \& Tritschler, A. 2002, A\&A, 394, 1069

von der Lühe, O. 1984, J. Opt. Soc. Am., 1, 510

Muller, R. 1977, Sol. Phys., 52, 249

Muller, R., \& Roudier, T. 1984, Sol. Phys., 94, 33

Muller, R., \& Mena, B. 1987, Sol. Phys., 112, 295

Nisenson, P., van Ballegooijen, A. A., de Wijn, A. G., \& Sütterlin, P. 2003, ApJ, 587, 458

Rimmele, T. R. 1994, A\&A, 290, 972

Sánchez Almeida, J., \& Lites, B. W. 2000, ApJ, 532, 1215

Sánchez Almeida, J., Asensio Ramos, A., Trujillo Bueno, J., \& Cernicharo, J. 2001, ApJ, 555, 978

Schlichenmaier, R. 2002, AN, 323, 303

Sheeley, N. R. 1969, Sol. Phys., 9, 347

Sobotka, M., Brandt, P. N., \& Simon, G. W. 1999, A\&A, 348, 621

Sobotka, M., \& Sütterlin, P. 2001, A\&A, 380, 714

Steiner, O., Hauschildt, P. H., \& Bruls, J. 2001, A\&A, 372, L13

Stenflo, J. 1973, Sol. Phys., 32, 41

Sütterlin, P. 2001, A\&A, 374, L21

Sütterlin, P., \& Wiehr, E. 1998, A\&A, 336, 367

Sütterlin, P., Wiehr, E., \& Stellmacher, G. 1999, Sol. Phys., 189, 57

Toenjes, K., \& Woehl, H. 1982, Sol. Phys., 75, 63

Vrabec, D. 1971, Solar Magnetic Fields, ed. R. Howard, IAU Symp., 43, 329

Weigelt, G. 1977, Optics Commun., 21, 55

Wiehr, E. 1978, A\&A, 69, 279

Wiehr, E. 1999, in Third Advances in Solar Physics Euroconference: Magnetic Fields and Oscillations, ASP Conf. Ser., 184, 86

Zhao, J., Kosovichev, A. G., \& Duvall, T. L. Jr. 2001, ApJ, 557, 384 